\title{
NONNULI DISTRIBUTION OF LRC FOR TESTING HOMOGENEITY OF COVARIANCE MATRICES OF COMPLETELY SYMMETRIC GAUSSIAN MODELS
}

\author{
A.K.GUPTA, D.K.NAGAR AND VIPIN TAYAL
}

\begin{abstract}
The nonnull moments of the likelihood ratio statistic for testing equality of covariance matrices of completely symmetric Gaussian models are obtained in terms of the Lauricella's hypergeometric functions and also in terms of zonal polynomials. Then the nonnull asymptotic distribution of the statistic is derived under certain alternatives for unequal samples.
\end{abstract}

\section{Introduction}

Let $\underset{\sim 1}{X}, \ldots, X_{m}$ be random vectors of order $p \times 1$ which are distributed independently as multivariate normal with mean vectors ${\underset{\sim}{1}}_{1}, \ldots,{ }_{\sim}^{\mu}$ and positive definite covariance matrices $\sum_{1}, \ldots, \sum_{m}$ respectively. Further, let $\sum_{i}^{1}=\sigma_{i}^{2}\left[\left(1-\rho_{i}\right) I_{p}+\rho_{i} e e_{\sim}^{\prime}\right]$ and $\mu_{i}=\mu_{i} e$, where $\sigma_{i}^{2}(>0), \rho_{i}$ and $\mu_{i}$ are unknown scalars, $i=1, \cdots, m$; Ip is the identity matrix of order $p$ and $e=(1, \cdots, 1)_{p \times 1}^{\prime}$. In the literature such a model is known as completely symmetric model.

Consider the null hypothesis

$$
H_{0}: \sum_{1}=\ldots=\sum_{m}=\sigma^{2}\left[(1-\rho) I_{p}+\rho e_{\sim} e^{\prime}\right]
$$

against the general alternative.

Krishnaiah and Pathak (1967), Han (1975) and Gupta and Nagar (1986. 1987) considered the problem of testing homogeneity of covariance matrices under the intraclass correlation model and have derived the null and nonull distributions of the test statistic (also see Gupta et al. 1975). Tayal et al. (1989) have derived the distribution of the likelihood ratio test statistic under $H_{0}$.

Received October 29, 1989, reviesed February 14, 1989.

AMS 1979 subject classification: Primary 62H10; Secondary 62H15.

Key words and phrases: Completely symmetric model; Nonnull moments; Zonal polynomials;

Generalized hypergeometric function; Distribution. 
In this paper we consider the problem of nonnull distribution. The nonnull moments of the likelihood ratio test statistic are derived in section 2 as a product of two Lauricella's hypergeometric functions, and also in series involving zonal polynomials when the sample sizes are equal. In section 3 and 4 , the asymptotic nonnull distribution of $-2 \ln \Lambda$ has been derived for certain alternatives.

\section{Likelihood ratio statistic and its moments.}

Suppose independent random samples $\underset{\sim}{X}{ }_{i 1}, \ldots, \underset{\sim}{X_{i N_{i}}}$ of size $N_{i}$ are taken form the $i^{\text {th }}$ population, $i=1, \cdots, m$. Let $Q$ be a $p \times p$ orthogonal maturix with first row $e_{\sim}^{\prime} / \sqrt{p}$, then $Q \sum_{i} Q^{\prime}=\operatorname{diag}\left(a_{i}, b_{i}, \cdots, b_{i}\right)=\sum_{i}^{*}$ and $\nu_{i}=Q \underset{\sim i}{\mu_{i}}=\mu_{i} Q e=\sqrt{p} \mu_{i} e_{1}$, say, where $a_{i}=\sigma_{i}^{2}\left[1+(p-1) \rho_{i}\right], b_{i}=\sigma_{i}^{2}\left(1-\rho_{i}\right)$ and ${\underset{\sim}{1}}_{1}=(1,0, \cdots, 0)_{p \times 1}^{\prime}$. The null hypothesis of testing homogeneity of covariance matrices is equivalent to $H_{0}: a_{1}=\ldots=a_{m}=a ; b_{1}=$ $\ldots=b_{m}=b$.

Let $\underset{\sim}{Y_{i u}}=Q \underset{\sim i u}{X_{i u}}, u=1, \cdots, N_{i}$, then $\underset{\sim i u}{Y_{i u}} \sim N\left(\nu_{i}, \sum_{i}^{*}\right)$. Further let, $W_{1 i}=\sum_{u=1}^{N_{i}}\left(y_{i 1 u}-\right.$ $\left.y_{i 1} \cdot\right)^{2}, W_{2 i}=\sum_{j=2}^{p} \sum_{u=1}^{N_{i}} y_{i j u}^{2}, W_{1}=\sum_{i=1}^{m} W_{1 i}$, and $W_{2}=\sum_{i=1}^{m} W_{2 i}$, where $y_{i 1} .=\frac{1}{N_{i}} \sum_{u=1}^{N_{i}} y_{i 1 u}, y_{i j u}$ being the $j^{\text {th }}$ component of ${\underset{\sim}{Y}}_{i u}$.

The likelihood ratio statistic can easily be written as (see Tayal et al. 1987),

$$
\Lambda=\Lambda_{1} \cdot \Lambda_{2}
$$

where

$$
\begin{aligned}
& \Lambda_{1}=\frac{N_{0}^{N_{0} / 2} \prod_{i=1}^{m} W_{1 i}^{N_{i} / 2}}{\prod_{i=1}^{m} N_{i}^{N_{i} / 2}\left(\sum_{i=1}^{m} W_{1 i}\right)^{N_{0} / 2}} \\
& \Lambda_{2}=\frac{\left[N_{0}(p-1)\right]^{N_{0}(p-1) / 2} \prod_{i=1}^{m} W_{2 i}^{N_{i}(p-1) / 2}}{\prod_{i=1}^{m}\left[N_{i}(p-1)\right]^{N_{i}(p-1) / 2}\left(\sum_{i=1}^{m} W_{2 i}\right)^{N_{0}(p-1) / 2}}
\end{aligned}
$$

and $N_{0}=\sum_{i=1}^{m} N_{i}$. Further, $\Lambda_{1}$ and $\Lambda_{2}$ are independent. We now derive two results giving the nonnull moments of $\Lambda_{1}$ and $\Lambda_{2}$ respectively.

Lemma 2.1. The $h^{\text {th }}$ nonnull moment of $\Lambda_{1}$ defined in (2.2) is given by

$$
E\left(\Lambda_{1}^{h}\right)=\left[\frac{N_{0}^{N_{0} / 2}}{\prod_{i=1}^{m} N_{i}^{N_{i} / 2}}\right]^{h} \cdot \frac{\Gamma\left[\left(N_{0}-m\right) / 2\right]}{\Gamma\left[\left\{N_{0}(1+h)-m\right\} / 2\right]}
$$




$$
\begin{aligned}
& \cdot \prod_{i=1}^{m}\left[\frac{\Gamma\left[N_{i}(1+h) / 2-1 / 2\right]\left(\eta_{1} a_{i}^{-1}\right)^{\left(N_{i}-1\right) / 2}}{\Gamma\left[\left(N_{i}-1\right) / 2\right]}\right] \\
& \cdot F_{D}^{(m)}\left(\frac{1}{2}\left(N_{0}-m\right) ; \frac{1}{2} N_{1}(1+h)-\frac{1}{2}, \cdots, \frac{1}{2} N_{m}(1+h)-\frac{1}{2}\right. \\
& \left.\quad \frac{1}{2} N_{0}(1+h)-\frac{1}{2} m ; 1-\eta_{1} a_{1}^{-1}, \cdots, 1-\eta_{1} a_{m}^{-1}\right)
\end{aligned}
$$

where $\left|1-\eta_{1} a_{i}^{-1}\right|<1, i=1, \cdots, m$ and $F_{D}^{(m)}$ is the Lauricella's hypergeometric function (Slater, 1966).

Proof. Since $W_{11} / a_{1}, \ldots, W_{1 m} / a_{m}$ are independent chi-squares with $N_{1}-1, \ldots$, $N_{m}-1$ d.f. respectively, we get the $h^{\text {th }}$ nonnull moment of $\Lambda_{1}$ as

$$
\begin{aligned}
E\left(\Lambda_{1}^{h}\right)= & {\left[\frac{N_{0}^{N_{0} / 2}}{\prod_{i=1}^{m} N_{i}^{N_{i} / 2}}\right]^{h} \int_{w_{11}>0} \cdots \int_{w_{1 m}>0} \prod_{i=1}^{m}\left[\frac{w_{1 i}}{\sum_{i=1}^{m} w_{1 i}}\right]^{N_{i} h / 2} } \\
& \cdot \prod_{i=1}^{m}\left[\frac{w_{1 i}^{\frac{1}{2}\left(N_{i}-1\right)-1} \exp \left\{-w_{1 i} / 2 a_{i}\right\}}{\left(2 a_{i}\right)^{\left(N_{i}-1\right) / 2} \Gamma\left[\left(N_{i}-1\right) / 2\right]}\right] d w_{11} \ldots d w_{1 m} .
\end{aligned}
$$

Replacing $\left[\sum_{i=1}^{m} w_{1 i}\right]^{-N_{0} h / 2}$ by an equivalent gamma integral. namely

$$
\Gamma\left(N_{0} h / 2\right) 2^{N_{0} h / 2}\left[\sum_{i=1}^{m} w_{1 i}\right]^{-N_{0} h / 2}=\int_{0}^{\infty} \exp \left[-\left(\sum_{i=1}^{m} w_{1 i}\right) x / 2\right] \cdot x^{N_{0} h / 2-1} d x
$$

for $\operatorname{Re}\left(N_{0} h / 2\right)>0$, changing the order of integration and integrating out $w_{11}, \ldots, w_{1 m}$, we have

$$
\begin{aligned}
E\left(\Lambda_{1}^{h}\right)= & {\left[\frac{N_{0}^{N_{0} / 2}}{\prod_{i=1}^{m} N_{i}^{N_{i} / 2}}\right]^{h}\left[\Gamma\left(N_{0} h / 2\right)\right]^{-1} \prod_{i=1}^{m}\left[\frac{\Gamma\left[N_{i}(1+h) / 2-1 / 2\right]}{a_{i}^{\left(N_{i}-1\right) / 2} \Gamma\left[\left(N_{i}-1\right) / 2\right]}\right] } \\
& \cdot \int_{0}^{\infty} x^{N_{0} h / 2-1} \prod_{i=1}^{m}\left(x+a_{i}^{-1}\right)^{-\left[N_{i}(1+h) / 2-1 / 2\right]} d x .
\end{aligned}
$$

Substituting $u=1 /\left(1+\eta_{1} x\right)$ in the above integrand and using (2.1) of Gupta and Nagar (1987), we can easily get (2.4).

Lemma 2.2. The $h^{\text {th }}$ nonnull moment of $\Lambda_{2}$ defined in (2.3) is given by

$$
\begin{aligned}
E\left(\Lambda_{2}^{h}\right)= & {\left[\frac{N_{0}^{N_{0} / 2}}{\prod_{i=1}^{m} N_{i}^{N_{i} / 2}}\right]^{h(p-1)} \frac{\Gamma\left[N_{0}(p-1) / 2\right]}{\Gamma\left[N_{0}(p-1)(1+h) / 2\right]} \prod_{i=1}^{m}\left[\frac{\Gamma\left[N_{i}(p-1)(1+h) / 2\right]\left(\eta_{2} b_{i}^{-1}\right)^{N_{i}(p-1) / 2}}{\Gamma\left[N_{i}(p-1) / 2\right]}\right] } \\
\cdot & F_{D}^{(m)}\left(\frac{1}{2} N_{0}(p-1) ; \frac{1}{2} N_{1}(p-1)(1+h), \cdots, \frac{1}{2} N_{m}(p-1)(1+h) ;\right. \\
& \left.\frac{1}{2} N_{0}(p-1)(1+h) ; 1-\eta_{2} b_{1}^{-1}, \cdots, 1-\eta_{2} b_{m}^{-1}\right)
\end{aligned}
$$


where $\left|1-\eta_{2} b_{i}^{-1}\right|<1, i=1, \cdots, m$.

Proof. In this case $W_{21} / b_{1}, \ldots, W_{2 m} / b_{m}$ are independent chi-squared variates with $N_{1}(p-1), \ldots, N_{m}(p-1)$ d.f. respectively. Therefore the $h^{t h}$ moment of $\Lambda_{2}$ can be obtained as in Lemma 2.1.

From Lemmas 2.1 and 2.2 and independence of $\Lambda_{1}$ and $\Lambda_{2}$ we obtain the following result.

Theorem 2.1. The $h^{\text {th }}$ nonnull moment of the test statistic $\Lambda$ defined by (2.1) is given by

$$
\begin{aligned}
E\left(\Lambda^{h}\right)= & \frac{N_{0}^{N_{0} p h / 2}}{\prod_{i=1}^{m} N_{i}^{N_{i} p h / 2}} \cdot \frac{\Gamma\left[\left(N_{0}-m\right) / 2\right] \Gamma\left[N_{0}(p-1) / 2\right]}{\Gamma\left[\left\{N_{0}(1+h)-m\right\} / 2\right] \Gamma\left[N_{0}(p-1)(1+h) / 2\right]} \\
& \cdot \prod_{i=1}^{m}\left[\frac{\Gamma\left[N_{i}(1+h) / 2-1 / 2\right]\left(\eta_{1} a_{i}^{-1}\right)^{\left(N_{i}-1\right) / 2}}{\Gamma\left[\left(N_{i}-1\right) / 2\right]} \frac{\left.\Gamma\left[N_{i}(p-1)(1+h) / 2\right]\left(\eta_{2} b_{i}^{-1}\right)^{N_{i}(p-1) / 2}\right]}{\Gamma\left[N_{i}(p-1) / 2\right]}\right] \\
& F_{D}^{(m)}\left(\frac{1}{2}\left(N_{0}-m\right) ; \frac{1}{2} N_{1}(1+h)-\frac{1}{2}, \cdots, \frac{1}{2} N_{m}(1+h)-\frac{1}{2}\right. \\
& \left.\frac{1}{2} N_{0}(1+h)-\frac{m}{2} ; 1-\eta_{1} a_{1}^{-1}, \cdots, 1-\eta_{1} a_{m}^{-1}\right) \\
& \cdot F_{D}^{(m)}\left(\frac{1}{2} N_{0}(p-1) ; \frac{1}{2} N_{1}(p-1)(1+h), \cdots, \frac{1}{2} N_{m}(p-1)(1+h) ;\right. \\
& \left.\frac{1}{2} N_{0}(p-1)(1+h) ; 1-\eta_{2} b_{1}^{-1}, \cdots, 1-\eta_{2} b_{m}^{-1}\right)
\end{aligned}
$$

where $\left|1-\eta_{1} a_{i}^{-1}\right|<1$ and $\left|1-\eta_{2} b_{i}^{-1}\right|<1, i=1, \cdots, m$.

When $N_{i}=N, i=1, \cdots, m$, the $h^{\text {th }}$ nonnull moment of the test statistic $\Lambda$ can be written in terms of zonal polynomials by substituting equivalent representation of Lauricella's hypergeometric function (see Gupta and Nagar, 1988a; eqn. 2.4) as

$$
\begin{aligned}
E\left(\Lambda^{h}\right)= & \frac{m^{m N p h / 2} \Gamma^{m}\left[\frac{N}{2}(1+h)-\frac{1}{2}\right] \Gamma^{m}\left[\frac{N(p-1)(1+h)}{2}\right]}{\Gamma\left[\frac{N-1}{2}\right] \Gamma^{m}\left[\frac{N(p-1)}{2}\right]} \\
& \cdot\left|\eta_{1} \Delta_{1}^{-1}\right|^{(N-1) / 2}\left|\eta_{2} \Delta_{2}^{-1}\right|^{N(p-1) / 2} \\
& \cdot \sum_{k=0}^{\infty} \sum_{j=0}^{\infty} \sum_{\mathcal{K}} \sum_{J}\left(\frac{1}{2} N(1+h)-\frac{1}{2}\right)_{\mathcal{K}}\left(\frac{N(p-1)(1+h)}{2}\right)_{J} \\
& \cdot \frac{C_{\mathcal{K}}\left(I_{m}-\eta_{1} \Delta_{1}^{-1}\right)}{k !} \cdot \frac{C_{j}\left(I_{m}-\eta_{2} \Delta_{2}^{-1}\right)}{j !} \\
& \cdot \frac{\Gamma\left[\frac{m}{2}(N-1)+k\right] \Gamma\left[\frac{m N(p-1)}{2}+j\right]}{\Gamma\left[\frac{m N(1+h)}{2}-\frac{m}{2}+k\right] \Gamma\left[\frac{m N(p-1)(1+h)}{2}+j\right]}
\end{aligned}
$$


where $\Delta_{1}=\operatorname{diag}\left(a_{1}, \ldots, a_{m}\right) ; \Delta_{2}=\operatorname{diag}\left(b_{1}, \ldots, b_{m}\right) ;\left\|I_{m}-\eta_{i} \triangle_{i}^{-1}\right\|<1 ; i=1,2$, and $C_{\mathcal{K}}(S)$ is a zonal polynomial (see James, 1964) of order $k$ in the latent roots of the $m \times m$ symmetric matrix $S ; \mathcal{K}=\left(k_{1}, \ldots, k_{m}\right), k_{1} \geq \cdots \geq k_{m} \geq 0, k_{1}+\ldots+k_{m}=k$.

\section{Asymptotic nomnull distribution for unequal samples}

In this section we derive the asymptotic nonnull distribution of a mutiple of $-2 \ln \Lambda$ when the sample sizes are unequal. Let $d_{i}=\frac{N_{i}}{N_{0}}, i=1, \cdots, m$,

$$
\delta_{0}=\frac{1}{12(m-1)}\left[\frac{(11 p-9)}{2(p-1)}\left(\sum_{i=1}^{m} d_{i}^{-1}-1\right)-\frac{3}{2}(m-1)(m+3)\right], \quad N_{0}=M_{0}+2 \delta_{0} .
$$

$N_{0} \tau=M_{0}$, and consider the alternative hypothesis

$$
A_{M_{0}}: 1-\eta_{1} a_{i}^{-1}=\frac{2 p_{i}}{d_{i} M_{0}} ; 1-\eta_{2} b_{i}^{-1}=\frac{2 q_{i}}{d_{i} M_{0}(p-1)}, i=1, \cdots, m .
$$

Under $A_{M_{0}}$, the characteristic function of $W=-2 \tau \ln \Lambda$ is derived from Theorem 2.1 as

$$
\begin{aligned}
\varphi_{W}(t)= & \varphi_{H_{0}}(t) \prod_{i=1}^{m}\left\{\left(1-\frac{2 p_{i}}{d_{i} M_{0}}\right)^{\frac{1}{2} M_{0} d_{i}+d_{i} \delta_{0}-\frac{1}{2}}\left(1-\frac{2 q_{i}}{d_{i} M_{0}(p-1)}{ }^{\left(d_{i} \delta_{0}+\frac{1}{2} M_{0} d_{i}\right)(p-1)}\right\}\right. \\
\cdot & F_{D}^{(m)}\left(\frac{1}{2} M_{0}+\delta_{0}-\frac{m}{2} ; \frac{1}{2} M_{0} d_{1} \theta+d_{1} \delta_{0}-\frac{1}{2}, \cdots, \frac{1}{2} M_{0} d_{m} \theta+d_{m} \delta_{0}-\frac{1}{2} ;\right. \\
& \left.\frac{1}{2} M_{0} \theta+\delta_{0}-\frac{m}{2} ; \frac{2 p_{1}}{d_{1} M_{0}}, \cdots, \frac{2 p_{m}}{d_{m} M_{0}}\right) \\
\cdot & F_{D}^{(m)}\left(\left(\frac{1}{2} M_{0}+\delta_{0}\right)(p-1) ;\left(\frac{1}{2} M_{0} d_{1} \theta+d_{1} \delta_{0}\right)(p-1)\left(\frac{1}{2} M_{0} d_{m} \theta+d_{m} \delta_{0}\right)(p-1) ;\right. \\
& \left.\left(\frac{1}{2} M_{0} \theta+\delta_{0}\right)(p-1) ; \frac{2 q_{1}}{d_{1} M_{0}(p-1)}, \cdots, \frac{2 q_{m}}{d_{m} M_{0}(p-1)}\right)
\end{aligned}
$$

where $\theta=1-2 \omega t, \omega=\sqrt{-1}$ and $\varphi_{H_{0}}(t)$ is the characteristic function of $-2 \tau \ln \Lambda$ when the null hypothesis is true and can be obtained from (3.1) of Tayal et al. (1989) by putting $h=-2 \omega \tau t$. The asymptotic expansion of $\varphi_{H_{0}}(t)$ is given below:

Lemma 3.1. Let $N_{i}=d_{i} N_{0}, d_{i}>0, i=1, \cdots, m$ with $\sum_{i=1}^{m} d_{i}=1$. When $H_{0}$ is true the characteristic function of $W=-2 \tau \ln \Lambda$, can be expanded as

$$
\varphi_{H_{0}}(t)=\theta^{-f / 2}\left[1+\frac{\gamma_{2}}{M_{0}^{2}}\left\{\theta^{-2}-1\right\}+O\left(M_{0}^{-3}\right)\right]
$$

where $f=2(m-1)$, and

$$
\gamma_{2}=\frac{1}{2}\left[\sum_{i=1}^{m} d_{i}^{-2}-\frac{m(m+1)(m+2)}{6}-2 f \delta_{0}^{2}\right] .
$$


Proof. See Anderson (1984, p. 311).

The asymptotic expansions of the Lauricella's hypergeometric functions involved in (3.2) are also given in the following two results:

Lemma 3.2. For $N_{i}=d_{i} N_{0}, d_{i}>0, i=1, \cdots, m$ with $\sum_{i=1}^{m} d_{i}=1$, the asymptotic expansion of $F_{D}^{(m)}$ involved in the characteristic function of $-2 \tau \ln \Lambda$ is

$$
\begin{aligned}
& F_{D}^{(m)}\left(\frac{1}{2} M_{0}+\delta_{0}-\frac{m}{2} ; \frac{1}{2} M_{0} d_{1} \theta+d_{1} \delta_{0}-\frac{1}{2}, \cdots, \frac{1}{2} M_{0} d_{m} \theta\right. \\
+ & \left.d_{m} \delta_{0}-\frac{1}{2} ; \frac{1}{2} M_{0} \theta+\delta_{0}-\frac{m}{2} ; \frac{2 p_{1}}{d_{1} M_{0}}, \cdots, \frac{2 p_{m}}{d_{m} M_{0}}\right) \\
= & \exp \left(\sum_{i=1}^{m} p_{i}\right)\left[1+\frac{1}{M_{0}}\left\{A_{0}+A_{1} \theta^{-1}\right\}+O\left(M_{0}^{-2}\right)\right]
\end{aligned}
$$

where

$$
A_{0}=\left(2 \delta_{0}-m\right)\left(\sum_{i=1}^{m} p_{i}\right)+\left(\sum_{i=1}^{m} p_{i}\right)^{2}
$$

and

$$
A_{1}=m\left(\sum_{i=1}^{m} p_{i}\right)-\left(\sum_{i=1}^{m} p_{i}\right)^{2}+\sum_{i=1}^{m}\left(p_{i}^{2} / d_{i}\right)-\sum_{i=1}^{m}\left(p_{i} / d_{i}\right) .
$$

Proof. Expanding $F_{D}^{(m)}$ in the series form using (2.2) of Gupta and Nagar (1987). one has

$$
\begin{aligned}
& F_{D}^{(m)}\left(\frac{1}{2} M_{0}+\delta_{0}-\frac{m}{2} ; \frac{1}{2} M_{0} d_{1} \theta+d_{1} \delta_{0}-\frac{1}{2}, \cdots ;\right. \\
& \left.\frac{1}{2} M_{0} d_{m} \theta+d_{m} \delta_{0}-\frac{1}{2} ; \frac{1}{2} M_{0} \theta+\delta_{0}-\frac{m}{2} ; \frac{2 p_{1}}{d_{1} M_{0}}, \cdots, \frac{2 p_{m}}{d_{m} M_{0}}\right) \\
= & \sum_{r=0}^{\infty} \sum_{R} G_{1}(R, t) \prod_{i=1}^{m}\left\{\left(\frac{2 p_{i}}{d_{i} M}\right)^{r_{i}} / r_{i} !\right\}
\end{aligned}
$$

where $R=\left(r_{1}, \ldots, r_{m}\right), r_{1}+\ldots+r_{m}=r, \sum_{R}$ denotes summation over all such partitions, and

$$
G_{1}(R, t)=\prod_{i=1}^{m}\left\{\left(\frac{1}{2} M_{0} d_{i} \theta+d_{i} \delta_{0}-\frac{1}{2}\right)_{r_{i}}\right\} \frac{\left(\frac{1}{2}\left(M_{0}-m\right)+\delta_{0}\right)_{r}}{\left(\frac{1}{2}\left(M_{0} \theta-m\right)+\delta_{0}\right)_{r}} .
$$

Expanding logarithm of $G_{1}(R, t)$, and converting back we get

$$
\begin{aligned}
G_{1}(R, t)= & \theta^{-r}\left[\prod_{i=1}^{m}\left(M_{0} d_{i} \theta / 2\right)^{r_{i}}\right]\left\{1+\sum_{i=1}^{m} \frac{r_{i}\left(2 d_{i} \delta_{0}+r_{i}-2\right)}{M_{0} d_{i} \theta}+O\left(M_{0}^{-2}\right)\right\} \\
& \cdot\left\{1+\frac{r\left(2 \delta_{0}-m+r-1\right)}{M_{0}}\left(1-\theta^{-1}\right)+O\left(M_{0}^{-2}\right)\right\} .
\end{aligned}
$$


Substituting $G_{1}(R, t)$ from (3.9) in (3.8), using multinomial summation formulae and summing over $r$ one can easily get (3.5).

Lemma 3.3. For $N_{i}=d_{i} N_{0}, d_{i}>0, i=1, \cdots, m$ with $\sum_{i=1}^{m} d_{i}=1$, the asymptotic expansion of $F_{D}^{(m)}$ involved in the characteristic function of $-2 \tau \ln \Lambda$ is

$$
\begin{aligned}
& F_{D}^{(m)}\left(\left(\frac{1}{2} M_{0}+\delta_{0}\right)(p-1) ;\left(\frac{1}{2} M_{0} d_{1} \theta+d_{1} \delta_{0}\right)(p-1), \cdots,\left(\frac{1}{2} M_{0} d_{m} \theta+d_{m} \delta_{0}\right)(p-1) ;\right. \\
& \left.\left(\frac{1}{2} M_{0} \theta+\delta_{0}\right)(p-1) ; \frac{2 q_{1}}{d_{1} M_{0}(p-1)}, \cdots, \frac{2 q_{m}}{d_{m} M_{0}(p-1)}\right) \\
= & \exp \left(\sum_{i=1}^{m} q_{i}\right) \cdot\left[1+\frac{1}{M_{0}}\left\{A_{0}^{\prime}+A_{1}^{\prime} \theta^{-1}\right\}+O\left(M_{0}^{-2}\right)\right]
\end{aligned}
$$

where

$$
A_{0}^{\prime}=2 \delta_{0}\left(\sum_{i=1}^{m} q_{i}\right)+\frac{1}{(p-1)}\left(\sum_{i=1}^{m} q_{i}\right)^{2}
$$

and

$$
A_{1}^{\prime}=\frac{1}{(p-1)}\left[\sum_{i=1}^{m}\left(q_{i}^{2} / d_{i}\right)-\left(\sum_{i=1}^{m} q_{i}\right)^{2}\right]
$$

Proof. Similar to the proof of Lemma 3.2.

Lemma 3.4. For large $M_{0}$, we have

$$
\begin{aligned}
& \prod_{i=1}^{m}\left\{\left(1-\frac{2 p_{i}}{d_{i} M_{0}}\right)^{\frac{1}{2} M_{0} d_{i}+d_{i} \delta_{0}-\frac{1}{2}}\left(1-\frac{2 q_{i}}{d_{i} M_{0}(p-1)}\right)^{\left(\frac{1}{2} M_{0} d_{i}+d_{i} \delta_{0}\right)(p-1)}\right\} \\
= & \exp \left[-\sum_{i=1}^{m}\left(p_{i}+q_{i}\right)\right] \cdot\left[1-\frac{1}{M_{0}}\left\{\frac{1}{(p-1)} \sum_{i=1}^{m} q_{i}\left\{2 \delta_{0}(p-1)+\frac{q_{i}}{d_{i}}\right\}\right.\right. \\
& \left.\left.+\sum_{i=1}^{m} p_{i}\left\{2 \delta_{0}+\frac{1}{d_{i}}\left(p_{i}-1\right)\right\}\right\}+O\left(M_{0}^{-2}\right)\right] .
\end{aligned}
$$

Proof. Expanding the logarithm of

$$
\prod_{i=1}^{m}\left\{\left(1-\frac{2 p_{i}}{d_{i} M_{0}}\right)^{\frac{1}{2} M_{0} d_{i}+d_{i} \delta_{0}-\frac{1}{2}}\left(1-\frac{2 q_{i}}{d_{i} M_{0}(p-1)}\right)^{\left(\frac{1}{2} M_{0} d_{i}+d_{i} \delta_{0}\right)(p-1)}\right\}
$$

and converting back, one can easily get (3.14).

Substituting (3.3), (3.5), (3.10) and (3.13) in (3.2) and inverting the resulting expansion of the characteristic function, one gets the following result: 
Theorem 3.1. The asymptotic expansion of the distribution of $W=-2 \tau \ln \Lambda$ under the alternative hypothesis stated in (3.1) is give by

$$
P[W \leq w]=P\left[\chi_{f}^{2} \leq w\right]+\frac{c}{M_{0}}\left\{P\left[\chi_{f+2}^{2} \leq w\right]-P\left[\chi_{f}^{2} \leq w\right]\right\}+O\left(M_{0}^{-2}\right)
$$

where

$$
C=-\left[\left(\sum_{i=1}^{m} \frac{p_{i}}{d_{i}}\right)-m\left(\sum_{i=1}^{m} p_{i}\right)+\left(\sum_{i=1}^{m} p_{i}\right)^{2}-\left(\sum_{i=1}^{m} \frac{p_{i}^{2}}{d_{i}}\right)+\frac{1}{p-1}\left(\left(\sum_{i=1}^{m} q_{i}\right)^{2}-\sum_{i=1}^{m}\left(\frac{q_{i}^{2}}{d_{i}}\right)\right)\right]
$$

and $\chi_{v}^{2}$ is the chi-square variate with $v$ d.f.

\section{Asymptotic momnull distribution for equal samples}

In this section we derive the asymptotic nonnull distribution of $-2 \ln \Lambda$ when the sample sizes are equal. Let $W=-(2 M / N) \ln \Lambda$ where $M=N-2 \delta(\delta$ is a constant to be specified later). Then the characteristic function $\varphi(t)$ of $W$ is

$$
\varphi(t)=\varphi_{1}(t) \cdot \varphi_{2}(t)
$$

where $\varphi_{1}(t)$ and $\varphi_{2}(t)$ are the characteristic functions of $-\left(\frac{2 M}{N}\right) \ln \Lambda_{1}$ and $-\left(\frac{2 M}{N}\right) \ln \Lambda_{2}$ respectively. In this section we derive the asymptotic distribution of $W$ by using the asymptotic expansion of $\varphi(t)$ as $M \rightarrow \infty$, for the following sequence of alternatives (see Khatri and Srivastava, 1974; Gupta and Nagar, 1987)

$$
\begin{aligned}
\text { (i) } I-\eta_{1} \Delta_{1}^{-1} & =\frac{2 P_{1}}{M} ; & I-\eta_{2} \Delta_{2}^{-1} & =\frac{2 P_{2}}{(p-1) M} \\
\text { (ii) } I-\eta_{1}^{-1} \Delta_{1} & =\frac{2 Q_{1}}{M} ; & I-\eta_{2} \Delta_{2}^{-1} & =\frac{2 P_{2}}{(p-1) M} \\
\text { (iii) } I-\eta_{1}^{-1} \Delta_{1} & =\frac{2 Q_{1}}{M} ; & I-\eta_{2}^{-1} \Delta_{2} & =\frac{2 Q_{2}}{(p-1) M} \\
\text { (iv) } I-\eta_{1} \Delta_{1}^{-1} & =\frac{2 P_{1}}{M} ; & I-\eta_{2}^{-1} \Delta_{2} & =\frac{2 Q_{2}}{(p-1) M}
\end{aligned}
$$

where $P_{1}, P_{2}, Q_{1}$ and $Q_{2}$ are fixed matrices.

Case (i):

$$
I-\eta_{1} \triangle_{1}^{-1}=\frac{2 P_{1}}{M} ; I-\eta_{2} \triangle_{2}^{-1}=\frac{2 P_{2}}{(p-1) M}
$$

Following the approximation procedure described in Gupta and Nagar (1987) (also see Khatri and Srivastava, 1974; Gupta and Nagar, 1988a 1988b) we obtain the asymptotic expansions for the characteristic functions $\varphi_{j}(t), j=1,2$ as follows:

$$
\begin{aligned}
\varphi_{j}(t)= & \theta^{-(m-1) / 2}\left[1+\frac{A^{(j)}}{M}\left(\theta^{-1}-1\right)\right. \\
& +\frac{1}{M^{2}}\left(B_{0}^{(j)}+B_{1}^{(j)} \theta^{-1}+B_{2}^{(j)} \theta^{-2}\right)+O\left(M^{3}\right), \quad j=1,2
\end{aligned}
$$


where

$$
\begin{aligned}
& A^{(1)}=-\left(\delta-\frac{1}{2}\right)(m-1)+\frac{m^{2}-1}{6 m}+\operatorname{tr} P_{1}^{2}-\frac{\left(\operatorname{tr} P_{1}\right)^{2}}{m} \\
& B_{0}^{(1)}=\frac{1}{2}\left[-\left(\delta-\frac{1}{2}\right)(m-1)+\frac{m^{2}-1}{6 m}+\operatorname{tr} P_{1}^{2}-\frac{\left(\operatorname{tr} P_{1}\right)^{2}}{m}\right]^{2}+r_{1} \\
& +\frac{4}{3}\left[\frac{\left(\operatorname{tr} P_{1}\right)^{3}}{m^{2}}-\operatorname{tr} P_{1}^{3}\right]+2\left(\delta-\frac{1}{2}\right)\left[\frac{\left(\operatorname{tr} P_{1}\right)^{2}}{m}-\operatorname{tr} P_{1}^{2}\right] \\
& B_{1}^{(1)}=-\left[\left\{-\left(\delta-\frac{1}{2}\right)(m-1)+\frac{m^{2}-1}{6 m}+\operatorname{tr} P_{1}^{2}-\frac{\left(\operatorname{tr} P_{1}\right)^{2}}{m}\right\}^{2}\right. \\
& +4\left(\delta-\frac{1}{2}\right)\left\{\frac{\left(\operatorname{tr} P_{1}\right)^{2}}{m}-\operatorname{tr} P_{1}^{2}\right\}+\frac{4\left(\operatorname{tr} P_{1}\right)}{m}\left[\frac{\left(\operatorname{tr} P_{1}\right)^{2}}{m}-\operatorname{tr} P_{1}^{2}\right] \\
& \left.+\frac{2}{m}\left[\frac{\left(\operatorname{tr} P_{1}\right)^{2}}{m}-\operatorname{tr} P_{1}^{2}\right]\right] \\
& B_{2}^{(1)}=-\left(B_{1}^{(1)}+B_{0}^{(1)}\right) \\
& r_{1}=\frac{2}{3 m^{2}}\left[-\frac{3}{2} m^{2}\left(\delta-\frac{1}{2}\right)^{2}(m-1)+\frac{1}{2} m\left(\delta-\frac{1}{2}\right)\left(m^{2}-1\right)\right] \\
& A^{(2)}=-\delta(p-1)(m-1)+\frac{m^{2}-1}{6 m}+\operatorname{tr} P_{2}^{2}-\frac{\left(\operatorname{tr} P_{2}\right)^{2}}{m} \\
& B_{0}^{(2)}=\frac{1}{2}\left[-\delta(p-1)(m-1)+\frac{m^{2}-1}{6 m}+\operatorname{tr} P_{2}^{2}-\frac{\left(\operatorname{tr} P_{2}\right)^{2}}{m}\right]^{2}+r_{2} \\
& +\frac{4}{3}\left[\frac{\left(\operatorname{tr} P_{2}\right)^{3}}{m^{2}}-\operatorname{tr} P_{2}^{3}\right]+2 \delta(p-1)\left[\frac{\left(\operatorname{tr} P_{2}\right)^{2}}{m}-\operatorname{tr} P_{2}^{2}\right] \\
& B_{1}^{(2)}=-\left[\left\{-\delta(p-1)(m-1)+\frac{m^{2}-1}{6 m}+\operatorname{tr} P_{2}^{2}-\frac{\left(\operatorname{tr} P_{2}\right)^{2}}{m}\right\}^{2}\right. \\
& +4 \delta(p-1)\left[\frac{\left(\operatorname{tr} P_{2}\right)^{2}}{m}-\operatorname{tr} P_{2}^{2}\right]+\frac{4\left(\operatorname{tr} P_{2}\right)}{m}\left\{\frac{\left(\operatorname{tr} P_{2}\right)^{2}}{m}-\operatorname{tr} P_{2}^{2}\right\} \\
& \left.+\frac{2}{m}\left\{\frac{\left(\operatorname{tr} P_{2}\right)^{2}}{m}-\operatorname{tr} P_{2}^{2}\right\}\right] \\
& B_{2}^{(2)}=-\left(B_{1}^{(2)}+B_{0}^{(2)}\right) \\
& r_{2}=\frac{2}{3 m^{2}}\left[-\frac{3}{2} m^{2} \delta^{2}(p-1)^{2}(m-1)+\frac{1}{2} m \delta(p-1)\left(m^{2}-1\right)\right] .
\end{aligned}
$$

Multiplying $\varphi_{1}(t)$ and $\varphi_{2}(t)$, we get the characteristic function $\varphi(t)$ as

$$
\varphi(t)=\theta^{-(m-1)}\left[1-\frac{C}{M}\left(\theta^{-1}-1\right)+\frac{1}{M^{2}}\left\{T_{0}+T_{1} \theta^{-1}+T_{2} \theta^{-2}\right\}+O\left(M^{-3}\right)\right]
$$

where

$$
\begin{aligned}
C= & -\left[-2 \delta(m-1)+\frac{1}{2}(m-1)+\frac{\left(m^{2}-1\right) p}{6 m(p-1)}+\left(\operatorname{tr} P_{1}^{2}\right)-\frac{\left(\operatorname{tr} P_{1}\right)^{2}}{m}\right. \\
& \left.+\frac{1}{p-1}\left(\left(\operatorname{tr} P_{2}^{2}\right)-\frac{\left(\operatorname{tr} P_{2}\right)^{2}}{m}\right)\right] .
\end{aligned}
$$


Now choose $\delta$ such that $-2 \delta(m-1)+\frac{1}{2}(m-1)+\frac{\left(m^{2}-1\right) p}{6 m(p-1)}=0$, giving $\delta=\frac{m(4 p-3)+p}{12 m(p-1)}$. For this value of $\delta, C, T_{0}, T_{1}$ and $T_{2}$ are given by

$$
\begin{aligned}
C= & C_{1}+\frac{C_{1}^{\prime}}{p-1} \\
T_{0}= & \frac{1}{2} C^{2}+\frac{4}{3} C_{2}+2\left(\delta-\frac{1}{2}\right) C_{1}+\frac{4}{3(p-1)^{2}} C_{2}^{\prime}+\frac{2 \delta}{(p-1)} C_{1}^{\prime}+r \\
T_{1}= & -\left\{C^{2}+4\left(\delta-\frac{1}{2}\right) C_{1}+\frac{4\left(\operatorname{tr} P_{1}\right)}{m} C_{1}+\frac{2}{m} C_{1}+\frac{4 \delta}{(p-1)} C_{1}^{\prime}\right. \\
& \left.\quad+\frac{4\left(\operatorname{tr} P_{2}\right)}{m(p-1)^{2}} C_{1}^{\prime}+\frac{2}{m(p-1)^{2}} C_{1}^{\prime}\right\} \\
T_{2}= & -\left(T_{0}+T_{1}\right)
\end{aligned}
$$

where $C_{1}=\frac{1}{m}\left(\operatorname{tr} P_{1}\right)^{2}-\left(\operatorname{tr} P_{1}^{2}\right), C_{1}^{\prime}=\frac{1}{m}\left(\operatorname{tr} P_{2}\right)^{2}-\left(\operatorname{tr} P_{2}^{2}\right), C_{2}=\frac{1}{m^{2}}\left(\operatorname{tr} P_{1}\right)^{3}-\left(\operatorname{tr} P_{1}^{3}\right)$, $C_{2}^{\prime}=\frac{1}{m^{2}}\left(\operatorname{tr} P_{2}\right)^{3}-\left(\operatorname{tr} P_{2}^{3}\right)$, and $r=2 \delta^{2}(m-1)-\frac{(m-1)(4 m+1)}{6 m}$.

Inverting (4.3), we get the asymptotic distribution of $W$ as

$$
\begin{aligned}
P[W \leq w]= & P\left[\chi_{f}^{2} \leq w\right]+\frac{C}{M}\left\{P\left[\chi_{f}^{2} \leq w\right]-P\left[\chi_{f+2}^{2} \leq w\right]\right\} \\
& +\frac{1}{M^{2}}\left\{T_{0} P\left[\chi_{f}^{2} \leq w\right]+T_{1} P\left[\chi_{f+2}^{2} \leq w\right]+T_{2}\left[\chi_{f+4}^{2} \leq w\right]\right\}+O\left(M^{-3}\right)
\end{aligned}
$$

where $f=2(m-1)$

Case (ii):

$$
I-\eta_{1}^{-1} \triangle_{1}=\frac{2 Q_{1}}{M} ; I-\eta_{2} \triangle_{2}^{-1}=\frac{2 P_{2}}{(p-1) M} .
$$

Replacing $P_{1}$ by $-Q_{1}\left(I+\frac{2 Q_{1}}{M}\right)$ in the coefficients in (4.4) and rearranging certain terms, various coefficients for this case are

$$
\begin{gathered}
C=C_{1}^{*}+\frac{1}{(p-1)} C_{1}^{\prime} \\
T_{0}=\frac{1}{2} C^{2}+2\left(\delta-\frac{1}{2}\right) C_{1}^{*}+\frac{8}{3} C_{2}^{*}-\frac{4}{m}\left(\operatorname{tr} Q_{1}\right) C_{1}^{*} \\
+\frac{4}{3(p-1)^{2}} C_{2}^{\prime}+\frac{2 \delta}{(p-1)} C_{1}^{\prime}+r \\
T_{1}=-\left\{C^{2}+4\left(\delta-\frac{1}{2}\right) C_{1}^{*}-\frac{8}{m}\left(\operatorname{tr} Q_{1}\right) C_{1}^{*}+\frac{2}{m} C_{1}^{*}\right. \\
\left.+4 C_{2}^{*}+\frac{4 \delta}{p-1} C_{1}^{\prime}+\frac{4\left(\operatorname{tr} P_{2}\right)}{m(p-1)^{2}} C_{1}^{\prime}+\frac{2}{m(p-1)^{2}} C_{1}^{\prime}\right\} \\
T_{2}=-\left(T_{0}+T_{1}\right),
\end{gathered}
$$

where

$$
C_{1}^{*}=\frac{1}{m}\left(\operatorname{tr} Q_{1}\right)^{2}-\operatorname{tr} Q_{1}^{2}, \quad C_{1}^{\prime}=\frac{1}{m}\left(\operatorname{tr} P_{2}\right)^{2}-\operatorname{tr} P_{2}^{2},
$$




$$
C_{2}^{* *}=\frac{1}{m^{2}}\left(\operatorname{tr} Q_{1}\right)^{3}-\operatorname{tr} Q_{1}^{3} \quad \text { and } \quad C_{2}^{\prime}=\frac{1}{m^{2}}\left(\operatorname{tr} P_{2}\right)^{3}-\operatorname{tr} P_{2}^{3}
$$

The asymptotic distribution in this case is given by

$$
\begin{aligned}
P[W \leq w]= & P\left[\chi_{f}^{2} \leq w\right]+\frac{C}{M}\left\{P\left[\chi_{f}^{2} \leq w\right]-P\left[\chi_{f+2}^{2} \leq w\right]\right\} \\
& +\frac{1}{M^{2}}\left\{T_{0} P\left[\chi_{f}^{2} \leq w\right]+T_{1} P\left[\chi_{f+2}^{2} \leq w\right]+T_{2} P\left[\chi_{f+4}^{2} \leq w\right]\right\}+O\left(M^{-3}\right)
\end{aligned}
$$

Case (iii):

$$
I-\eta_{1}^{-1} \Delta_{1}=\frac{2 Q_{1}}{M} ; I-\eta_{2}^{-1} \Delta_{2}=\frac{2 Q_{2}}{(p-1) M}
$$

The asymptotic distribution is given by (4.5) by replacing $P_{2}$ by $-Q_{2}\left(I+\frac{2 Q}{(p-1) M}\right)$. Now the coefficients for this case are given by

$$
\begin{gathered}
C=C_{1}^{*}+\frac{1}{(p-1)} C_{1}^{* \prime} \\
T_{0}=\frac{1}{2}\left[C_{1}^{*}+\frac{1}{(p-1)} C_{1}^{* \prime}\right]^{2}+2\left(\delta-\frac{1}{2}\right) C_{1}^{*}-\frac{4}{m}\left(\operatorname{tr} Q_{1}\right) C_{1}^{*}+\frac{8}{3} C_{2}^{*} \\
+\frac{2 \delta}{p-1} C_{1}^{* \prime}-\frac{4\left(\operatorname{tr} Q_{2}\right)}{m(p-1)^{2}} C_{1}^{* \prime}+\frac{8}{3(p-1)^{2}} C_{2}^{* \prime}+r \\
T_{1}=-\left\{\left[C_{1}^{*}+\frac{1}{(p-1)} C_{1}^{* \prime}\right]^{2}+4\left(\delta-\frac{1}{2}\right) C_{1}^{*}-\frac{8}{m}\left(\operatorname{tr} Q_{1}\right) C_{1}^{*}+\frac{2}{m} C_{1}^{*}+4 C_{2}^{*}\right. \\
\left.+\frac{4 \delta}{(p-1)} C_{1}^{* \prime}-\frac{8\left(\operatorname{tr} Q_{2}\right)}{m(p-1)^{2}} C_{1}^{* \prime}+\frac{2}{m(p-1)^{2}} C_{1}^{* \prime}+\frac{4}{(p-1)^{2}} C_{2}^{* \prime}\right\} \\
T_{2}=-\left(T_{0}+T_{1}\right)
\end{gathered}
$$

where $C_{1}^{*}=\frac{1}{m}\left(\operatorname{tr} Q_{1}\right)^{2}-\operatorname{tr} Q_{1}^{2}, C_{1}^{* \prime}=\frac{1}{m}\left(\operatorname{tr} Q_{2}\right)^{2}-\operatorname{tr} Q_{2}^{2}$,

$$
C_{2}^{*}=\frac{1}{m^{2}}\left(\operatorname{tr} Q_{1}\right)^{3}-\operatorname{tr} Q_{1}^{3}, \quad \text { and } \quad C_{2}^{* \prime}=\frac{1}{m^{2}}\left(\operatorname{tr} Q_{2}\right)^{3}-\operatorname{tr} Q_{2}^{3} .
$$

Case (iv):

$$
I-\eta_{1} \triangle_{1}^{-1}=\frac{2 P_{1}}{M} ; I-\eta_{2}^{-1} \triangle_{2}=\frac{2 Q_{2}}{(p-1) M}
$$

The asymptotic distribution is given by (4.4) by replacing $P_{2}$ by $-Q_{2}\left(I+\frac{2}{M(p-1)} Q_{2}\right)$ where now,

$$
C=C_{1}+\frac{1}{(p-1)} C_{1}^{* \prime}
$$




$$
\begin{aligned}
T_{0}= & \frac{1}{2}\left[C_{1}+\frac{1}{(p-1)} C_{1}^{* \prime}\right]^{2}+\frac{4}{3} C_{2}+2\left(\delta-\frac{1}{2}\right) C_{1}+r+\frac{2 \delta}{(p-1)} C_{1}^{* \prime} \\
& -\frac{4}{m(p-1)^{2}}\left(\operatorname{tr} Q_{2}\right) C_{1}^{* \prime}+\frac{8}{3(p-1)^{2}} C_{2}^{* \prime} \\
T_{1}= & -\left\{\left[C_{1}+\frac{1}{(p-1)} C_{1}^{* \prime}\right]^{2}+4\left(\delta-\frac{1}{2}\right) C_{1}+\frac{4\left(\operatorname{tr} P_{1}\right)}{m} C_{1}+\frac{2}{m} C_{1}+\frac{4 \delta}{(p-1)} C_{1}^{* \prime}\right. \\
& \left.-\frac{8\left(\operatorname{tr} Q_{2}\right)}{m(p-1)^{2}} C_{1}^{* \prime}+\frac{2}{m(p-1) 2} C_{1}^{* \prime}+\frac{4}{(p-1)^{2}} C_{2}^{* \prime}\right\}
\end{aligned}
$$

$T_{2}=-\left(T_{0}+T_{1}\right)$ and $C_{1}, C_{1}^{* \prime}, C_{2}, C_{2}^{* \prime}$ and $r$ are defined above.

\section{References}

[1] Anderson, T. W. An Introduction to Multivariate Statistical Analysis, 2nd Edition. John Wiley, New York, (1984).

[2] Gupta, A. K. Chattopadhyay, A. K. and Krishnaiah, P. R. "Asymptotic distribution of the determinant of some random matrices", Comm. Statist., 4, (1975) 33-47.

[3] Gupta, A. K. and Nagar, D. K. "Testing equality of covariance matrices under intraclass correlation structure", Tamkang J. Math., 17(4), (1986) 7-16.

[4] Gupta, A. K. and Nagar, D. K. "Nonnull distribution of the likelihood ratio criterion for testing equality of covariance matrices under intraclass correlation model", Comm. Statist. Theory-Meth. 16(1987), 3323-3341.

[5] Gupta, A. K. and Nagar, D. K. "Asymptotic expansion of the nonnull distribution of likelihood ratio statistic for testing multisample sphericity", Comm. Statist. Theory-Meth. 17, (1988a) 3145-3156.

[6] Gupta, A. K. and Nagar, D. K. "Nonnull distribution of likelihood ratio criterion for testing multisample sphericity in the complex case", Aust. J. Statist. 30, (1988b) 307-318.

[7] Han, C. P. "Testing the equality of covariance matrices under intraclass correlation models", Ann. Inst. Statist. Math., 27, (1975) 349-356.

[8] James, A. T. "Distribution of matrix variates and latent rocts deived from normal samples", Ann. Math. Statist., 35, (1964) 475-501.

[9] Khatri, C. G. and Srivastava , M. S. "Asymptotic expansions of the nonnull distributions of likelihood ratio criteria for covariance matrices", Ann. Statist., 2, (1974) 109-117.

[10] Krishnaiah, P. R. and Pathak, P. K. "Tests for the equality of covariance matrices under the intraclass correlation model", Ann. Math. Statist., 38, (1967) 1286-1288.

[11] Slater, L. J. Generalized Hypergeometric Functions. Cambridge University Press, London, (1966).

[12] Tayal, Vipin, Gupta, A. K. and Nagar, D. K. "Testing homogeneity of covariances matrices of completely symmetric Gaussian models," Metron, 47, (1989), 334-346.

Bowling Green State University and University of Rajasthan. 